\title{
THE CLOSED NEIGHBORHOOD AND FILTER CONDITIONS IN SOLID SEQUENCE SPACES
}

\author{
P.D. JOHNSON, JR.
}

Department of Algebra, Combinatorics, and Analysis

120 Math Annex

Auburn University

Auburn, Alabama, 36849, U.S.A.

(Received June 30, 1988)

ABSTRACT. Let $E$ be a topological vector space of scalar sequences, with topology $\tau$; $(E, \tau)$ satisfies the closed neighborhood condition iff there is a basis of neighborhoods at the origin, for $\tau$, consisting of sets which are closed with respect to the topology $\pi$ of coordinate-wise convergence on $E ;(E, \tau)$ satisfies the filter condition iff every filter, Cauchy with respect to $\tau$, convergent with respect to $\pi$, converges with respect to $\tau$.

Examples are given of solid (definition below) normed spaces of sequences which (a) fail to satisfy the filter condition, or (b) satisfy the filter condition, but not the closed neighborhood condition. (Robertson and others have given examples fulfilling (a), and examples fulfilling (b), but these examples were not solid, normed sequence spaces.) However, it is shown that among separated, separable solid pairs $(E, \tau)$, the filter and closed neighborhood conditions are equivalent, and equivalent to the usual coordinate sequences constituting an unconditional Schauder basis for $(E, \tau)$. Consequently, the usual coordinate sequences do constitute an unconditional Schauder basis in every complete, separable, separated, solid pair $(E, \tau)$.

KEY WORDS AND PHRASES. Topological vector space, sequence space, norm, Banach space, separated, separable, solid, filter condition, closed neighborhood condition, unconditional Schauder basis, Abschnitt-Konvergenz (AK).

1980 AMS SUBJECT CLASSIFICATION CODE. 46 A45.

1. BACKGROUND AND DEFINITIONS.

Suppose that $E$ is a topological vector space under two topologies, $\tau$ and $\pi$. Following Robertson [1] and Garling [2], we shall say that (E, $\tau$ ) satisfies the closed neighborhood condition (with respect to $\pi$ ) if and only if there is a base of $\tau$-neighborhoods of the origin which are $\pi$-closed, and that $(E, \tau)$ satisfies the filter 
condition (with respect to $\pi$ ) if and only if each filter in $E$, Cauchy with respect to $\tau$, and convergent with respect to $\pi$, converges with respect to $\tau$. (More exactly, Robertson might say that $(E, \tau)$ satisfies the filter condition with respect to $\pi$ and the identity injection of $(E, \tau)$ into $(E, \pi)$.

Observe that if $(E, \tau)$ is complete, then $(E, \tau)$ trivially satisfies the filter condition, with respect to any $\pi$. Although the result will not be used much here, a theorem of Robertson [1], Theorem 1) is worth noting: if (E, $\pi$ ) is separated (Hausdorf $f$ ), and $\tau$ is finer than $\pi$, then the completion of (E, $\tau$ ) is naturally embedded in the completion of $(E, \pi)$ if and only if $(E, \tau)$ satisfies the filter condition, with respect to $\pi$.

The importance of the closed neighborhood condition arises from a result of Bourbaki ([3], Proposition 8, Chap. 1., 1), which may also be found in Treves [4] (Lemma 34.2); this result is approximately Proposition 10 of [1], which we restate here.

LEMMA 1.1. If $(E, \pi)$ is separated, $\tau$ is finer than $\pi$, and $(E, \tau)$ satisfies the closed neighborhood condition, then $(E, \tau)$ satisfies the filter condition.

In this paper $E$ will be a subspace of $\omega$, the vector space of all scalar sequences (the scalars may be efther the real or complex numbers); $\pi$ will be the topology of coordinate-wise convergence on $E$. This is the relative topology on $E$ induced by the product topology on $w$, thought of as a countable product of copies of the scalar field.

If $x \varepsilon w$, the solid hull of $x$ is

$$
S(x)=\left\{\begin{array}{llll}
y & \varepsilon & \omega & \left|y_{n}\right|<\left|x_{n}\right| \text { for al1 } n
\end{array}\right\} \text {. }
$$

A subset of $\omega$ is solid if and only if it contains the solid hull of each of its elements. Throughout, $E$ will be solid. A topology $\tau$ on $E$ (with which $E$ becomes a t.v.s.) will be called solid if there is a neighborhood base at the origin for $\tau$ consisting of solid sets. When both $E$ and $\tau$ are solid, we will refer to $(E, \tau)$ as a solid pair.

Note that if a norm $\rho$ on $E$ satisifes

$$
\text { y } \varepsilon S(x) \subseteq E \text { imp1ies that } \rho(y)<\rho(x) \text {, }
$$

and $E$ is solid, then the norm topology on $E$ associated with $\rho$ is solid. Conversely, if $E$ is solid, any solid norm topology on $E$ is associable with such a norm (since the convex hull of a solid set is solid); when $\rho$ satisfies ( 1.1$) \rho$ will be called a solid norm.

LEMMA 1.2. Suppose that $E$ is solid, and $\rho$ is a solid norm on $E$. Let $\tau$ denote the topology on $E$ defined by $\rho$, and let $U=\{x \in E ; \rho(x)<1\}$. Then $(E, \tau)$ satisfies the closed neighborhood condition if and only if the closure of $U$ in ( $E, \pi$ ) is bounded in $(E, \tau)$. 
PROOF. If the closure $W$ of $U$ in $(E, \pi)$ is bounded in $(E, \tau)$, then $\{r W ; r>0\}$ is a neighborhood base at the origin in ( $E, \tau)$ consisting of sets closed in ( $E, \pi)$. If, on the other hand, $(E, \tau)$ satisifes the closed neighborhood condition, then the closure in $(E, \pi)$ of some set $r U, r>0$, is contained in $U$; thus $W \subseteq r^{-1} U$.

The coordinate projections on $E$ are the functionals $f_{n}$ defined by

$$
\mathrm{f}_{\mathrm{n}}(\mathrm{x})=\mathrm{x}_{\mathrm{n}} \cdot
$$

The following lemma is well known. A proof of the equivalence of (a) and (c) appears in Johnson and Mohapatra [5] (Prop. 2.5).

LEMMA 1.3. Suppose that $(E, \tau)$ is a solid pair. The following are equivalent.

(a) The coordinate projections are continuous on $E$.

(b) $\tau$ is finer than $\pi$.

(c) $(E, \tau)$ is separated.

The coordinate sequences are the sequences $e_{n} \varepsilon \omega$ defined by $e_{n}(m)=1$ if $n=$ $m, e_{n}(m)=0$ otherwise. We shall assume throughout that $E$ contains the coordinate sequences. The finite sections are the functions $P_{n}: E \rightarrow E$ defined by

$P_{n}(x)=\sum_{k<n} x_{k} e_{k}$. Following the terminology in Kothe [6], if for each $x \in E$, the sequence $\left(P_{n}(x)\right)$ converges to $x$ in

$(E, \tau)$, we will say that $(E, \tau)$ is $A K$ (for Abschnitt-Konvergenz). When (E, $\tau$ ) is a separated solid pair, $(E, \tau)$ is $A K$ if and only if the coordinate sequences form a Schauder basis for $(E, \tau)$; the solidity of $E$ and $\tau$ then guarantees that the basis is unconditional.

The following is Proposition 2.9 of [5].

LEMMA 1.4. Suppose that $(E, \tau)$ is an AK solid pair. Then $(E, \tau)$ satisfies the closed neighborhood condition.

In the next section we give two examples of solid, solidly normed spaces $E \subseteq \omega ;$ the first fails to satisfy the filter condition, and the second satisfies the filter condition, but not the closed neighborhood condition. The object is to augment the supply of such examples; see Lindenstrauss and Tzafrifi [7], Garling [2], and Gaposhkin and Kadets ([8]). As far as we know, these are the first known (or, in the case of Example 2.1, noticed) such examples in which (E, $\tau$ ) is a solid, solidly normed sequence space, and $\pi$ is the topology of coordinate-wise convergence on $E$.

The norm topology in Example 2.1 is not consistent in the sense of Ruckle [9]; by Theorem 1 of [2] it could not possibly be. The norm in Example 2.2 is consistent.

In section 3 it is shown that among separable, separated solid pairs (E, $\tau$ ), the filter and closed neighborhood conditions are equivalent, and are equivalent to (E, $\tau$ ) being AK. As an easy consequence, it follows that in every complete separable separated solid pair $(E, \tau)$, the coordinate sequences form an unconditional Schauder basis of $(E, \tau)$. (Thus, the famous separable Banach spaces with no Schauder basis, 
while possibly realizable as sequence spaces, are not realizable as solid, solidly normed sequence spaces.)

In Section 4 we discuss a problem mentioned in [5], still unresolved, related to the results of this paper, and one other problem that arises from these results.

2. EXAMPLES.

EXAMPLE 2.1. Let $E=\left\{x \in \omega ;\left(n x_{n}\right)\right.$ is bounded $\}$. Let $\rho$ be defined by

$$
\rho(x)=\left(\sum\left|x_{n}\right|^{2}\right)^{1 / 2}+11 m_{n} \sup \left|n x_{n}\right|
$$

Clearly $E$ is solid, and $\rho$ is a solid norm on $E$. Let $x^{(k)} \in E$ be defined by

$$
\begin{aligned}
& x_{n}^{(k)}=n^{-1} \text { if } n>k, \\
& x_{n}^{(k)}=0 \text { if } n<k .
\end{aligned}
$$

Then $\left(x^{(k)}\right)_{k}$ is Cauchy with respect to $\rho$, converges to zero in $(E, \pi)$, and $\rho\left(x^{(k)}\right)>1$ for all k; thus, by Lemma 1.3, $\left(x^{(k)}\right)$ cannot converge in $(E, \tau)$, $\tau$ the norm topology associated with $\rho$. Consequently, $(E, \tau)$ does not satisfy the filter condition.

EXAMPLE 2.2. Partition the positive integers into infinite sets $\mathrm{J}_{1}, \mathrm{~J}_{2}, \ldots$. For each $n$, define $p_{n}$ on $\ell_{\infty}$ by

$$
p_{n}(x)=\sup _{k \in J}\left|x_{k}\right|+n \lim _{k \in J_{n}}\left|x_{k}\right|
$$

Let $E=\left\{x \in \ell_{\infty}: \sum_{n=1}^{\infty} p_{n}(x)<\infty\right\}$ and define $\rho$ on $E$ by

$$
\rho(x)=\sum_{n=1}^{\infty} p_{n}(x) \text {. }
$$

Clearly $E$ is solid and $\rho$ is a solid norm on $E$; $E$ is an " $\ell_{1}$ direct sum" of the spaces $\ell_{\infty}\left(J_{n}\right)$, each isomorphic to $\ell_{\infty}$, so $E$, with $\rho$, is complete. Consequently, (E, $\tau$ ) satisfies the filter condition, where, as usual, $\tau$ is the topology determined by $\rho$.

For each $n$, let $g_{n}$ denote the characteristic sequence of $J_{n}$; i.e. $g_{n}(m)=1$ if m $\varepsilon J_{n}$, and $g_{n}(m)=0$ otherwise. Note that $\rho\left(g_{n}\right)=n+1$ for each $n$. Also, $\rho\left(P_{k}\left(g_{n}\right)\right)<1$ for each $k$, and $g_{n}$ is the limit, in $(E, \pi)$, of $\left(P_{k}\left(g_{n}\right)\right)_{k}$. By Lemma 1.2 , $(E, \tau)$ does not satisfy the closed neighborhood condition.

3. ON SEPARABLE SOLID PAIRS.

THEOREM 3.1. Suppose that $(E, \tau)$ is a separable, separated solid pair. The following are equivalent.

(a) $(E, \tau)$ satisfies the filter condition.

(b) (E, $\tau$ ) satisfies the closed neighborhood condition.

(c) $(\mathrm{E}, \tau)$ is $\mathrm{AK}$.

PROOF. By Lemma 1.3 and 1.1 , (b) implies (a), and (c) implies (b) by Lemma 1.4. 
What remains to be shown is that (a) implies (c).

Suppose that $(E, \tau)$ is not AK. Then there is some $x \varepsilon E$ such that the sequence $\left(P_{n}(x)\right)$ does not converge to $x$ in $(E, \tau)$. But $\left(P_{n}(x)\right)$ does converge to $x$ in $(E, \pi)$; therefore, if (a) holds, it must be that $\left(P_{n}(x)\right)$ is not Cauchy in $(E, \tau)$. Therefore, there is a solid $\tau$-neighborhood $U$ of the origin in $E$ and two sequences $\left(n_{t}\right),\left(m_{t}\right)$ of positive integers satisfying

and

$$
n_{t}<m_{t}<n_{t+1}
$$

$$
P_{m_{t}}(x)-P_{n_{t}}(x) \notin U, t=1,2, \cdots
$$

If $t<r$ then $n_{t}<m_{t}<n_{r}$, so $P_{m_{t}}(x)-P_{n_{t}}(x) \varepsilon s\left(P_{n_{r}}(x)-P_{n_{t}}(x)\right)$; consequently, $P_{n_{r}}(x)-P_{n_{t}}(x) \notin U$ for $r>t$, because $P_{m_{t}}(x)-P_{n_{t}}(x) k U$ and $U$ is solid.

For each $t$, let

$$
B_{t}=\left\{n_{t}+1, \ldots, n_{t+1}\right\}
$$

For each set $T$ of positive integers, let $y^{(T)} \varepsilon \omega$ be defined by

$$
\begin{aligned}
& y_{n}^{(T)}=x_{n} \text { if } n \in \bigcup_{t \varepsilon T}^{B_{t}}, \\
& y_{n}^{(T)}=0 \text { otherwise. }
\end{aligned}
$$

Each $y^{(T)}$ is in the solid hull of $x$, so $y^{(T)} \varepsilon E$ for each $T$. If $T_{1} \neq T_{2}$ are two sets of positive integers, then the solid hull of $\mathrm{y}^{\left(\mathrm{T}_{1}\right)}-\mathrm{y}^{\left(\mathrm{T}_{2}\right)}$ contains $P_{n_{t+1}}(x)-P_{n_{t}}(x) \varepsilon U$, for some $t$; consequently $y^{\left(T_{1}\right)}-y^{\left(T_{2}\right)} \varepsilon U$.

Suppose $V$ is a balanced, open $\tau$-neighborhood of the origin in $E$ satisfying $V+V \subseteq U$. The paragraph preceding implies that $\{y(T)+V ; T$ is a set of positive integers $\}$ is an uncountable collection of mutually disjoint topen non-empty subsets of $E$; consequently $(E, \tau)$ is not separable. We have arived at this conclusion assuming (a) and not (c). Consequently, (a) implies (c), when (E, $\tau$ ) satisfies the hypothesis of the theorem.

COROLLARY 3.1. If $(E, \tau)$ is a complete separable, separated solid pair, then $(E, \tau)$ is $A K$.

PROOF. If $(E, \tau)$ is complete, then $(E, \tau)$ satisfies the filter condition.

It has been pointed out to the author that the hard part of the theorem above, (a) implies (c), resembles a corollary of a well known result about Banach lattices ([7], Proposition 1.a.7). Since the scalars may be the complex numbers here, it would 
be reckless to deduce the theorem from the Banach lattice result, even in the special case in which $(E, \tau)$ is a Banach space, but the point is well made; the author admits to having rediscovered an argument and a result already in the literature, in a somewhat different setting. The author is of the opinion that such rediscoveries, if recognized as such, do no harm, and may serve to stimulate interest in the original.

\section{REMARKS AND PROBLEMS.}

Suppose $(E, \tau)$ is a solid pair and $A=\left(a_{m n}\right)$ is an infinite matrix with nonnegative entries and no zero columns. Following the notation of Johnson and Mohapatra $[5,10,11]$, we set

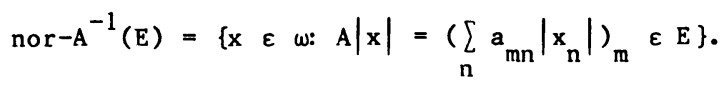

Then nor $-A^{-1}(E)$ is a solid subspace of $\omega$; if we require each column of $A$ to be in $E$, then nor $-A^{-1}(E)$ contains the coordinate sequences; further, nor-A $A^{-1}(E)$ is naturally equipped with a solid t.v.s. topology, nor-A $A^{-1}(\tau)$, obtained by taking as a neighborhood base at the origin, the sets

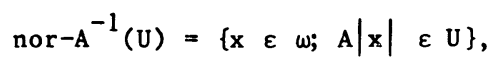

as $U$ ranges over solid $t$-neighborhoods of the origin in $E$.

In [5] it is shown that $\left(\operatorname{nor}-A^{-1}(E)\right.$, nor $\left.-A^{-1}(\tau)\right)$ will inherit a great deal from $(E, \tau)$ (the property of being $A K$, for instance), but there seems to be a mysterious difficulty regarding completeness. In [5] (Theorem 2.13) and [11] (Prop. $1.1)$ it is shown that (nor $-A^{-1}(E)$, nor $-A^{-1}(\tau)$ ) is complete when $(E, \tau)$ is, provided either that $(E, \tau)$ satisfies the closed neighborhood condition, or that the rows of $A$ are finite sequences. (In [11], A is lower triangular, but this condition can be relaxed.) Richard Haydon and Mireille Levy have since shown the author, in a private communication, that these provisions can be omitted when (E, $\tau$ ) is a Banach space, and their proof extends to the cases in which $(E, \tau)$ is locally convex and metrizable. In general, however, the problem remains unresolved.

PROBLEM 4.1. Suppose $(E, \tau)$ is a separated, complete solid pair, and $A$ is an infinite matrix with non-negative entries, no zero columns, and every column in $E$. Is $\left(\right.$ nor $-A^{-1}(E)$, nor-A $\left.{ }^{-1}(\tau)\right)$ necessarily complete?

The remarks preceding say that if a counterexample can be found, then $A$ will have to have some rows (indeed, infinitely many rows) with infinitely many non-zero entries, and $(E, \tau)$ will not be locally convex and metrizable.

It is shown in [5] (Theorem 2.10) that if (E, $\tau$ ) satisfies the closed neighborhood condition, then so does (nor-A $\left.A^{-1}(E), \operatorname{nor}-A^{-1}(\tau)\right)$.

PROBLEM 4.2. Suppose that $(E, \tau)$ and $A$ are as in Problem 4.1, except that (E, $\tau$ ) is not necessarily complete. Suppose that $(E, \tau)$ satisfies the filter condition. Does 
$\left(\right.$ nor $-A^{-1}(E)$, nor $\left.-A^{-1}(\tau)\right)$ necessarily satisfy the filter condition?

Problems 4.1 and 4.2 are equivalent in the following sense. Suppose that $C$ is a class of separated solid pairs, with the property that if $(E, \tau) \varepsilon C$ and $(E, \tau)$ satisfies the filter condition, then the completion $(\hat{E}, \hat{\tau})$ of $(E, \tau)$ also is an element of C. (By Robertson's Theorem $1([1]),(\hat{E}, \hat{\tau})$ will be a sequence space; it is straightforward to see that $(\hat{E}, \hat{\tau})$ will be a solid pair). If the answer to the question in 4.1 or 4.2 is yes, whenever $(E, \tau)$ is confined to $C$, then the answer to the question in the other is yes, whenever $(E, \tau)$ is in $C$ (with $A$ fixed, or allowed to range over some selection of matrices). Thus, for instance, the result of Haydon and Levy mentioned above implies that when $(E, \tau)$ is locally convex, metrizable, and satifies the filter condition, and A satisfies the hypothesis of Problem 4.1 with respect to $(E, \tau)$, then (nor- $A^{-1}(E)$, nor $-A^{-1}(\tau)$ ) satisfies the filter condition. For another instance, the answer to 4.2 is yes whenever the rows of $A$ are finite sequences.

We omit the proof of the equivalence. In one direction the proof uses the theorem of Robertson mentioned above, and the fact that the completion of a solid pair is a solid pair, when 1 is a sequence space. In the other direction the proof resembles that of Theorem 2.13 of [5].

Finally, we note a problem left unresolved by the results of sections 2 and 3 .

PROBLEM 4.3. Does there exist a separable, separated solid pair (E, $\tau$ ), preferably normed, which does not satisfy the filter condition?

The space in Example 2.1 is not separable. To see this, let $\left.\mathbb{K}_{r} ; r \varepsilon c\right\}$ be a collection of infinite sets of positive integers indexed by the continuum $c$ with the property that $r_{1} \neq r_{2}$ implies that $K_{r_{1}} n_{r_{2}}$ is finite. (Such a collection may be obtained by putting the positive integers in one-to-one correspondence with the rational numbers, and for each real number $r$, taking $K_{r}$ to be the elements of a sequence of distinct rationals convergent to $\left.r_{0}\right)$ For each $r \varepsilon c$, let $x^{(r)} \varepsilon \omega$ be defined by $x_{n}^{(r)}=1 / n$ if $n \varepsilon K_{r}, x_{n}^{(r)}=0$ otherwise. Then, with $E$ and $\rho$ as in Example $2.1,\left\{x^{(r)}: r \in c\right\}$ is an uncountable collection of elements of $E$, with $\rho\left(x^{\left(r_{1}\right)}-x^{\left(r_{2}\right)}\right)>1$ for $r_{1} \neq r_{2}$.

The theorem of section 3 bears on Problem 4.3; it suffices to find a separable, separated solid pair $(E, \tau)$ which is not AK. The proof of that theorem says more; for each $x \in E$, the sequence $\left(P_{n}(x)\right)$ will have to be Cauchy.

\section{ACKNOWLEDGEMENT .}

Besides Richard Haydon and Mireille Levy, whose invaluable (to me) contribution is mentioned earlier in this section, I would like to thank R.N. Mohapatra, Stan Rajnak, and M.S. Ramanujan for various helpful and illuminating remarks on the subject of this paper. 


\section{REFERENCES}

1. ROBERTSON, W., Completions of topological vector spaces, Proc. London Math. Soc. (3) 8 (1958), 242-257.

2. GARLING, D.J.H., The filter condition, the closed neighborhood condition, and consistent seminorms, Duke J. 38 (1971), 299-303.

3. BOURBAKI, N., Elements de Mathematique, livre V: Espaces vectoriels topologiques Act. Scien. et Ind., nos. 1189,1229, Hermann, Paris !953-5.

4. TREVES, R., Topological Vector Spaces, Distributions, and Kernels, Academic Press, New York - London, 1967.

5. JOHNSON, P.D., Jr. and MOHAPATRA, R.N., The maximal normal subspace of the inverse image of a normal space of sequences by a non-negative matrix transformation, Annales Polonici Math. XLV (1985), 105-120.

6. KOTHE, G., Topological Vector Spaces I., translation by D.J.H. Garling, SpringerVerlag, New York, 1969.

7. LINDENSTRAUSS, J. and TZAFRIRI, L., Classical Banach Spaces II, SpringerVerlag, Berlin-Heidelberg-New York, 1979.

8. GAPOSHKIN, V.F. and KADETS, M.J., Operator bases in Banach spaces, Math. Sb. N.S. 61 (103), 1963, 5-12.

9. RUCKLE, W., Lattices of sequence spaces, Duke J. 35 (1968), 481-503.

10. JOHNSON, P.D., Jr. and MOHAPATRA, R.N., Inequalities involving infinite matrices with non-negative entries, General Inequalities 2, ISNM 47, Birkhauser Verlag, Basel, 1980, 55-80.

11. JOHNSON, P.D., Jr. and MOHAPATRA, R.N., Inequalities involving lower triangular matrices, Proc. London Math. Soc. XLI (1980), 83-137. 


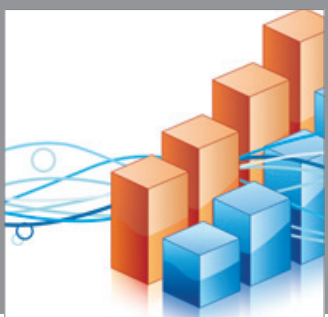

Advances in

Operations Research

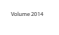

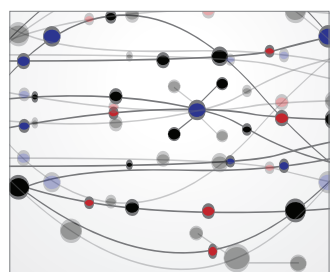

\section{The Scientific} World Journal
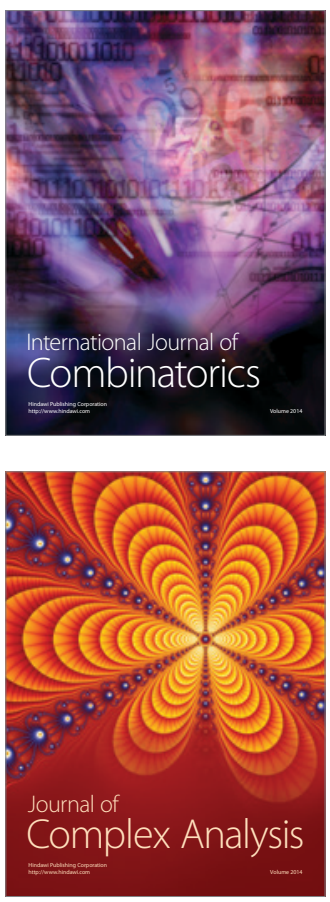

International Journal of

Mathematics and

Mathematical

Sciences
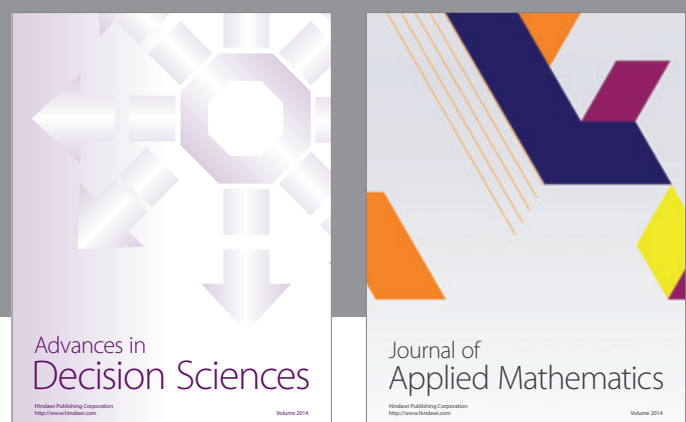

Journal of

Applied Mathematics
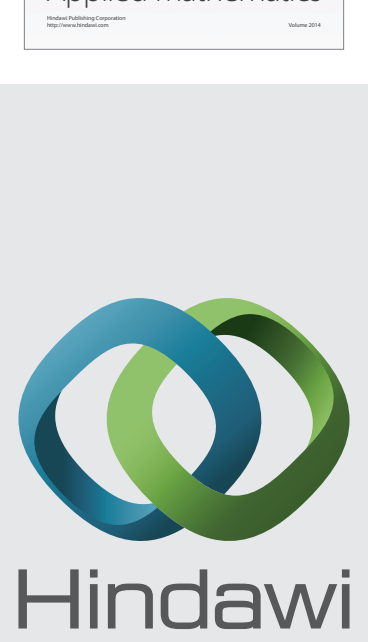

Submit your manuscripts at http://www.hindawi.com
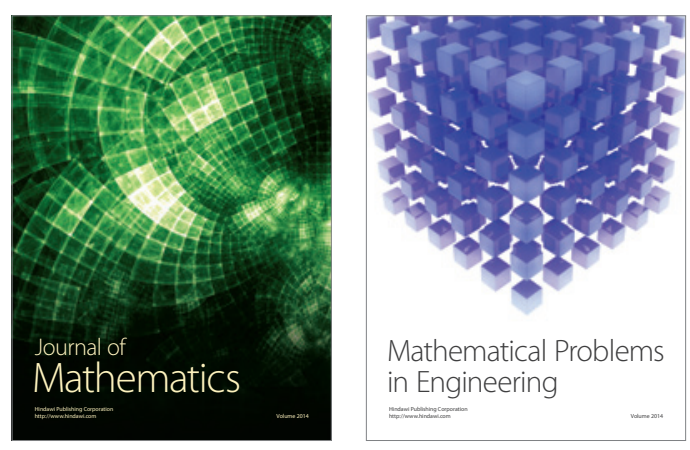

Mathematical Problems in Engineering
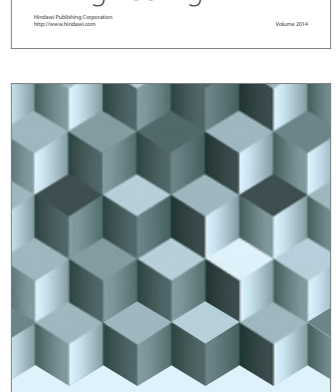

Journal of

Function Spaces
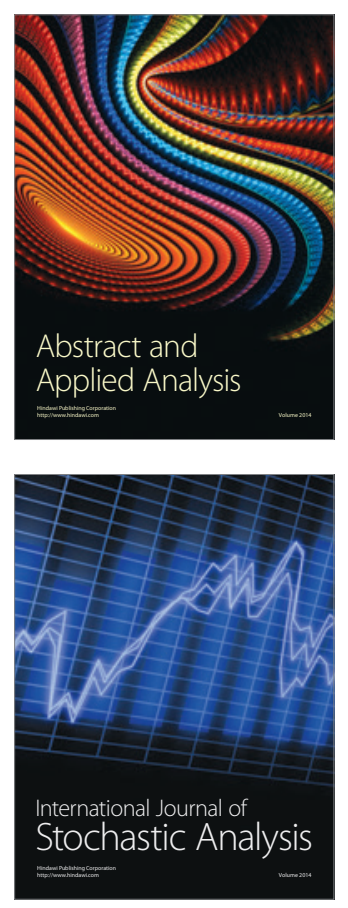

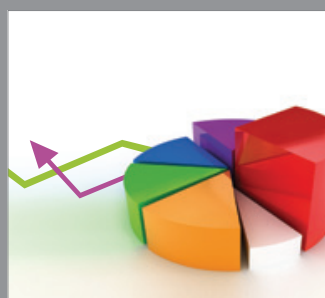

ournal of

Probability and Statistics

Promensencen
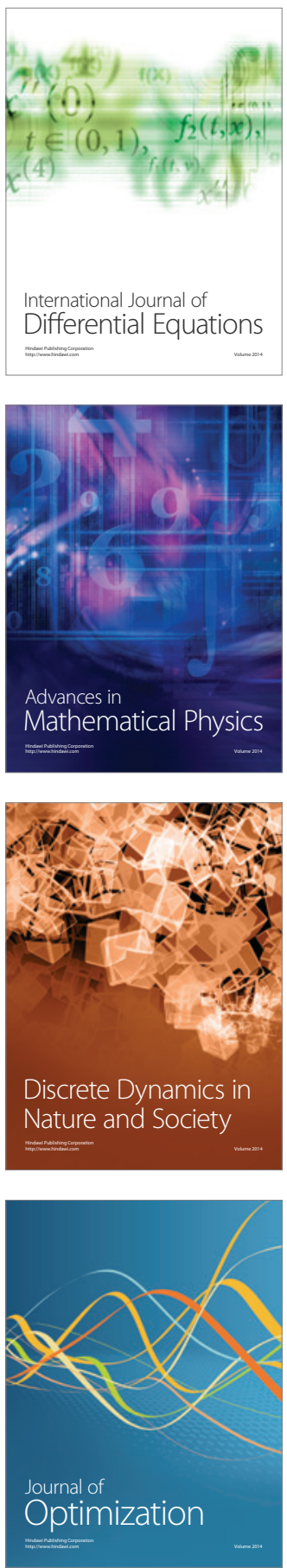